\title{
Desarrollo de un Alimento para Mascotas Utilizando Subproductos de la Industria Atunera en la Cuidad de Manta
}

\author{
Ligia Elizabeth Paredes Peñafiel, \\ Docente de Tiempo Completo de la Facultad de Mecánica, \\ Escuela Superior Politécnica de Chimborazo (ESPOCH), Ecuador \\ Danny Daniel De La A Peredo, Investigador \\ Universidad Agraria del Ecuador Facultad de Ciencias Agrarias

\section{Tatiana Sánchez Herrera, MsC \\ Vinicio Paredes Peralta, $\mathrm{MsC}$ \\ Fredy Erazo Rodríguez, $\mathrm{MsC}$ \\ María González Cabrera, $\mathrm{MsC}$} \\ Docentes de Tiempo Completo de la Facultad de Ciencias Pecuarias, \\ Escuela Superior Politécnica de Chimborazo (ESPOCH), Ecuador
}

Doi: 10.19044/esj.2019.v15n18p63 ＵRL:http://dx.doi.org/10.19044/esj.2019.v15n18p63

\section{Resumen}

La presente investigación tiene como propósito dar una alternativa al aprovechamiento de estos excedentes del atún; elaborando un alimento para mascota, garantizando sus propiedades físico-químicas y nutricionales. Para lo cual se analizó sensorialmente el efecto del porcentaje del excedente de atún en la formulación al $(50 \%, 70 \%$ y 90\%). Los datos obtenidos de la prueba sensorial fueron analizados usando el programa Statgraphics Centurion XVI. Se realizó la prueba ANOVA y Pruebas de Múltiples Rangos para determinar diferencias significativas entre formulaciones con un nivel del $95 \%$ de confianza. La evaluación sensorial demostró que la mejor formulación para el alimento de mascota con excedente del atún fue la del $90 \%$ de excedente de atún, ya que fue la de mayor calificación tanto en el olor, color y textura dada por los panelista y además fue el de mayor agrado por las mascotas en la prueba de degustación.

Palabras clave: Excedentes, Atún, Alimentos para mascotas 


\title{
Development of Pet Food by Using Products of the Tuna Industry in the City of Manta
}

\author{
Ligia Elizabeth Paredes Peñafiel,
}

Docente de Tiempo Completo de la Facultad de Mecánica, Escuela Superior Politécnica de Chimborazo (ESPOCH), Ecuador

Danny Daniel De La A Peredo, Investigador

Universidad Agraria del Ecuador Facultad de Ciencias Agrarias

Tatiana Sánchez Herrera, $M s C$

Vinicio Paredes Peralta, $\mathrm{MsC}$

Fredy Erazo Rodríguez, $\mathrm{MsC}$

María González Cabrera, $\mathrm{MsC}$

Docentes de Tiempo Completo de la Facultad de Ciencias Pecuarias, Escuela Superior Politécnica de Chimborazo (ESPOCH), Ecuador

\begin{abstract}
The present investigation has as purpose to give an alternative to the tuna surplus; by elaborating a pet food, thats meet the physical-chemical standards and nutritional properties. For which a sensorial test was done, to analize the effect of the percentage of the surplus in the formulation of $50 \%$, $70 \%$ and $90 \%$. The data obtained from the sensory test were analyzed using the Statgraphics Centurion XVI program. The ANOVA and Multiple Range Tests were performed to determine significant differences between formulations, with a level of $95 \%$ confidence. The sensory evaluation showed that the best formulation for the tuna surplus pet food was that of $90 \%$, since it was the one with the highest qualification in terms of odor, color and texture given by the panelists and it was also the one with greater liking.
\end{abstract}

Keywords: Surplus, Tuna, Pet food

\section{Introduction}

La industria alimenticia es la encargada de transformar la materia prima en productos terminados, se caracteriza por ser la más antigua en el mercado y que tiene un mayor impacto ambiental, debido a las deficiencias que existen en la actualidad por parte de la legislación. Por otro lado, la actividad pesquera en general genera altos ingresos para el país por ende la cadena alimenticia no acaba sólo en alimentos para el consumo humano sino 
que quiere incursionar en la elaboración de alimentos para mascotas con los excedentes del atún.

El atún se encuentra en todos los mares intertropicales del mundo, pero su captura se da principalmente en el Atlántico y en el Pacifico cuando migra de sur a norte; debido a que es durante esta migración que el atún nada en aguas más superficiales que se encuentren a $10^{\circ} \mathrm{C}$ (Hramann, 2010). Por su parte, la producción y exportación del atún en el país se la considera como una actividad tradicional; a pesar de su novedad es un aportante significativo al ingreso, a la generación de empleos y a la creación de valor.

Esta actividad en el Ecuador cuenta con dos localidades importantes que han permitido por sus condiciones y ubicaciones geográficas, el desarrollo de esta industria; las cuales son: Manta (Manabí) y Posorja (parroquia rural de Guayaquil). En ambas localidades se concentra la industria atunera ecuatoriana (Calderón, 2014).

Por su parte, la industria atunera es una rama industrial que hace más de 4 décadas conlleva un serio impacto ambiental no sólo dentro del país, sino en el mundo entero. Entre las deficiencias de las actividades pesqueras dentro del país está la escasa aplicación de la legislación que protege las especies marinas y el ambiente.

Sin embargo, la preocupante situación sobre el ambiente en los últimos tiempos ha adquirido una gran importancia, no se debe únicamente al hecho de que en la actualidad se esté creando conciencia, sino también al alto nivel de desperdicios en el atún en el proceso de enlatado, por esta razón es mejor buscar alternativas para lograr mejoras en los procesos productivos, reduciendo desperdicios en los pescados en proceso. Por tal razón el objetivo de esta invetigación consiste en aprovechar al máximo los excedentes del atún, haciendo énfasis en el desarrollo de un alimento para mascotas por su capacidad de aprovechamiento, que sería factible para maximizar recursos de las empresas atuneras.

\section{Metodología}

Se desarrolló un estudio con un enfoque experimental a través de la práctica para el desarrollo de un alimento para mascotas utilizando los excedentes de atún en la ciudad de Manta. Los dos tipos de investigación que se utilizaron fueron: el método deductivo que permitió investigar las potenciales causas que intervienen en el problema y el método analítico estudió de las diferentes causas y hechos. La observación de estas causas permitió determinar el efecto que incide en el desarrollo de un alimento para mascota. 


\section{Tratamientos}

Se desarrollaron tres tratamientos en los cuales se realizaron variaciones en las cantidades de sus ingredientes. El primer tratamiento se consideraron los excedentes del atún al $50 \%$, en el segundo al $70 \%$ y en el último tratamiento al $90 \%$.

Tabla 1. Tratamientos para la elaboración de un alimento de mascotas con excedentes de atún. (De la A, et al. 2017)

\begin{tabular}{lccc}
\hline \multicolumn{1}{c}{ Ingrediente } & $\begin{array}{c}\text { Excedentes del } \\
\text { Atún al 50\% } \\
\text { (T1) }\end{array}$ & $\begin{array}{c}\text { Excedentes del } \\
\text { Atún al 70\% } \\
\text { (T2) }\end{array}$ & $\begin{array}{c}\text { Excedentes del } \\
\text { Atún al 90\% } \\
\text { (T3) }\end{array}$ \\
\hline Excedente de atún & 21,5 & 30,1 & 38,7 \\
Carne de res molida & & & \\
cocida & 21,5 & 12,9 & 4,3 \\
Almidón de yuca & 10,0 & 10,0 & 10,0 \\
Aislado proteico de & & 5,0 & 5,0 \\
soya & 5,0 & & \\
Sal & 3,0 & 3,0 & 3,0 \\
Ajo en polvo & 3,0 & 3,0 & 3,0 \\
Agua & 25,0 & 25,0 & 25,0 \\
Zanahoria & 10,0 & 10,0 & 10,0 \\
Sorbato de Potasio & 0,4 & 0,4 & 0,4 \\
Ac Cítrico & 0,6 & 0,6 & 0,6 \\
Total & $100 \%$ & $100 \%$ & $100 \%$ \\
\hline
\end{tabular}

2. $\quad$ Elaboración del proceso de alimento para mascotas con excedentes del atún

El proceso de elaboración del alimento para mascotas con excedente de atún se realizó en la planta piloto de la Facultad de Ciencias Agrarias de la Universidad Agraria del Ecuador.

Se recibió el excedente del atún (vísceras, cola, y carne negra), luego se procedió a la limpieza los cuales fueron colocadas en tinas para someterlas a un proceso de lavado donde son liberados y purificados de cualquier material orgánico e inorgánico que lo puedan contaminar. El excedente entra a un proceso de picado con el fin de obtener una mayor uniformidad en el tratamiento térmico a la cual se sometió.

\section{3. $\quad$ Formulación base del alimento para mascot}

La formulación del alimento de mascotas se desarrolló considerando dos aspectos: En primer lugar, los ingredientes más comunes en los alimentos de mascotas comercializados actualmente en el Ecuador: carne de res, pollo, hígado de res, proteína texturizada de soya, harina de trigo, harina de soya, almidón de maíz, agua, azúcar, sal, ajo en polvo, extracto de cebolla, sulfato de calcio, ácido fosfórico, sorbato de potasio (como conservante), ácido cítrico (como conservante), nitrito de sodio (para retener el color). 
El segundo aspecto que se tomó en cuenta fueron los ingredientes presentes en el alimentos para mascotas comercializados en Ecuador (Dog chow y michu), productos con características similares a las deseadas en el nuevo producto: carne de res, harina de soya, proteína texturizada de soya, azúcar, grasa, queso cheddar en polvo, agua, gelatina, almidón modificado, suero de leche, color caramelo, sal, ácido cítrico, sabores naturales y artificiales, sorbato de potasio, lecitina.

Tabla 2. Formulación de Alimento para Mascota. (De la A, et al. 2017)

\begin{tabular}{lr}
\hline Ingrediente & g/100g producto \\
\hline Excedente de atún & 25,0 \\
Carne de res molida cocida & 18,0 \\
Almidón de yuca & 10,0 \\
Aislado proteico de soya & 5,0 \\
Sal & 3,0 \\
Ajo en polvo & 3,0 \\
Agua & 25,0 \\
Zanahoria & 10,0 \\
Sorbato de Potasio & 0,4 \\
Ácido Cítrico & 0,6 \\
& \\
\hline Total & $\mathbf{1 0 0}$
\end{tabular}

\section{Requisitos específicos para alimento completo para felinos}

Se consideraron los parámetros establecidos en la norma Venezolana COVENIN 1888-83 alimento completo para caninos y felinos. De acuerdo a este documento, un alimento para gatos debe contener los componentes que se especifican en la tabla 3.

Tabla 3. Requisitos específicos para felinos. (COVENIN, 2017)

\begin{tabular}{lllll}
\hline \multirow{2}{*}{ COMPONENTES } & \multicolumn{3}{c}{ TIPO DE ALIMENTO } \\
& $\begin{array}{l}\text { Granulado } \\
\text { y expandido }\end{array}$ & Semi-húmedo & Enlatado & $\begin{array}{l}\text { METODO DE } \\
\text { ENSAYO }\end{array}$ \\
\hline Humedad, \% máx & 12,5 & 35,0 & 75,0 & COVENIN 1156 \\
Proteína, \% mín & 30,0 & 22,0 & 8,5 & COVENIN 1195 \\
Grasa, \% mín & 6,0 & 4,5 & 1,7 & COVENIN 1162 \\
Fibra, \% máx & 6,0 & 4,5 & 1,7 & COVENIN 1194 \\
Calcio, \% máx & 2,0 & 1,3 & 0,5 & COVENIN 986 \\
Fósforo, \% mín & 0,8 & 0,5 & 0,2 & COVENIN 1178 \\
\hline
\end{tabular}

Salmonella spp. Deberá estar ausente en 5 muestras de $25 \mathrm{~g}$ determinada según la norma (COVENIN1291, 2017).

\section{Análisis sensorial}

En el análisis sensorial se evaluaron las preferencias referentes a 3 atributos (Color, Olor y Textura). Para esto se usaron las pruebas hedónicas, las cuales declaran el nivel de agrado o desagrado de los jueces por medio de una escala verbal-numérica. Usualmente, esta escala va desde "me gusta 
muchísimo", pasando por "no me gusta ni me disgusta" hasta "me disgusta muchísimo".

Las pruebas sensoriales de aceptación indicaron si los productos con sus distintas formulaciones son aceptados o rechazados por parte del consumidor. La prueba se llevó a cabo con una escala estructurada de 5 puntos, siendo las alternativas de respuesta las siguientes: "Me disgusta mucho" (1 puntos) "Me disgusta un poco (2 puntos) "ni me gusta, ni me disgusta" (3 puntos) "me gusta un poco" (4 puntos) y "Me gusta mucho" (5 puntos).

En el ensayo se realizaron pruebas con humanos y también con gatos en la ciudad de Guayaquil y La Libertad, donde se les entregaron las muestras y se les explicaron cómo llenar la prueba con sus resultados; se codificaron las muestras asignándoles una numeración como se especifica en la tabla 4. Asimismo, fue aplicada la prueba de degustación a los gatos, para observar cuál de las 3 formulaciones eran aceptados favorablemente por las mascotas. En la cual se colocaron las 3 muestras con el fin de identificar cuál fue la de mayor consumo.

Tabla 4. Codificación de las muestras

\begin{tabular}{cc}
\hline CÓDIGO & Porcentajes de Excedentes de atún \\
\hline 354 & $50 \%$ \\
417 & $70 \%$ \\
598 & $90 \%$ \\
\hline
\end{tabular}

6. $\quad$ Análisis estadístico

Para el análisis estadístico del alimento para mascota, se utilizó el programa Statgraphics Centurion XVI (Statistical Graphics Corporation, UK).

De los resultados obtenidos los cuales presentan diferencias significativas entre las muestras se inició a elaborar una comparación de rangos múltiples de Kruskal-Wallis.

\section{Resultados}

Dentro de la provincia de Manabí se encuentra la ciudad de Manta donde más se concentra la industria atunera ya que se ubica en una posición estratégica, su posición en el punto más saliente de la costa sudamericana de Pacífico con un puerto natural de aguas profundas y un puerto nacional de primer orden. (Fueltala P. , 2013).

El manejo inadecuado de los residuos sólidos no peligrosos, no solo representa riesgos y posibles efectos potenciales para la salud humana, sino que además contribuye a la generación de contaminación en los recursos agua, suelo y aire. Por tal motivo se desarrolló una nueva alternativa para el uso de estos desechos (excedente de atún), minimizando residuos de los procesos productivos en la industrialización del atún reduciendo el impacto ambiental, 
dando un punto favorable al nivel económico de la industria con la innovación y elaboración de un subproducto.

\section{Resultados de los análisis sensoriales}

Para las diferentes formulaciones se calificó el nivel de agrado de 3 atributos (Color, Olor y Textura) en una escala hedónica de 5 puntos.

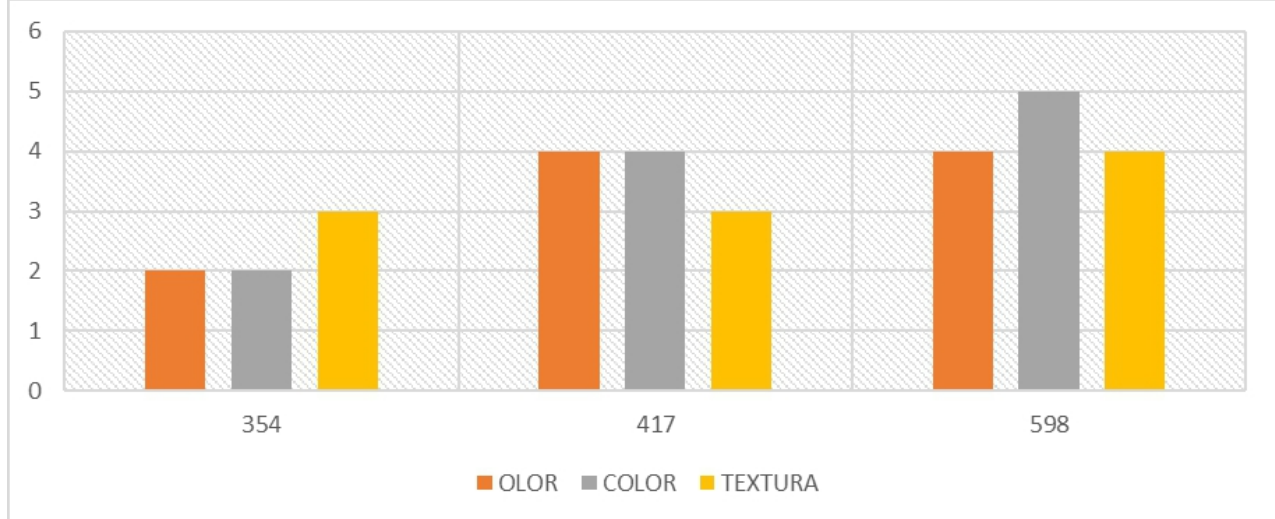

Figura 1. Comparación sensorial. (De la A, et al. 2017) 354: Tratamiento 1, Excedente de Atún 50\%; 417: Tratamiento 2, Excedente de Atún 70\%; 598 Tratamiento 3, Excedente de Atún $90 \%$.

En la figura 1 se observa que la muestra con mayor porcentaje de excedente de atún (90\%) fue la que obtuvo una mayor calificación en la escala hedónica. Para las formulaciones con menor porcentaje de excedente $(50 \%$ y $70 \%)$, los encuestados le dieron calificación más baja.

Según los resultados de los panelistas se consideraron que las muestras con mayor porcentaje de excedente fueron las mejores en cuanto a evaluación sensorial. Se puede decir que a niveles superiores al $70 \%$ de excedente de atún en la formulación hubo una mayor aceptación del producto por parte de los panelistas.

Una vez realizada las encuestas a las mascotas (gatos) se logró determinar el grado de aceptabilidad al tratamiento $3(90 \%)$, que fue formulado partiendo de los componentes integrados en la tabla 2 . Con respecto a la prueba de degustación de las mascotas los resultados reflejan que el tratamiento 3 tuvo una mayor aceptación con un 53\%. Por otro lado la formulación con menor porcentaje de excedente (tratamiento 1) obtuvo menos aceptación con un 13\%. En este sentido la figura 2 muestra los resultados de las pruebas de degustación la cual se realizó a las mascotas. 


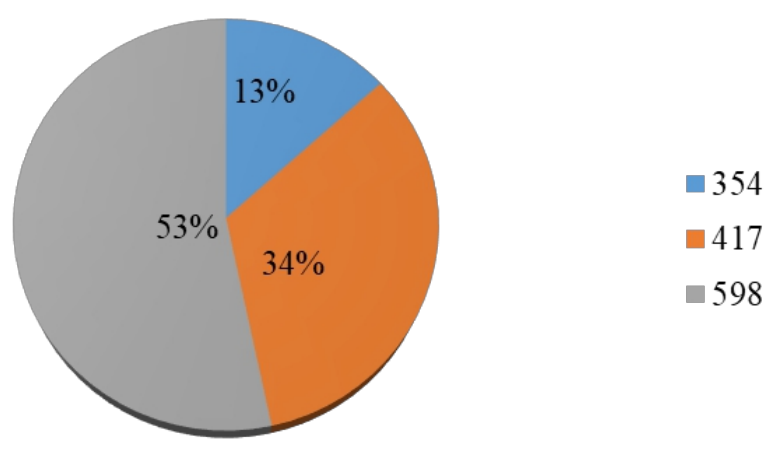

Figura 2. Resultado de prueba de degustación aplicado a mascotas. 354: Tratamiento 1, Excedente de Atún 50\%; 417: Tratamiento 2, Excedente de Atún 70\%; 598 Tratamiento 3, Excedente de Atún $90 \%$

\section{Análisis estadísticos pruebas sensoriales}

En la tabla 5 se muestran los resultados de la evaluación sensorial de las diferentes muestras.

Tabla 5: Evaluación sensorial de las formulaciones del alimento para mascotas con respecto al porcentaje del excedente del atún. (De la A, et al. 2017)

\begin{tabular}{llll}
\hline CÓDIGO & OLOR & COLOR & TEXTURA \\
\hline $\mathbf{3 5 4}$ & $2,5 \pm 0,9 \mathrm{a}$ & $2,3 \pm 0,7 \mathrm{a}$ & $2,6 \pm 0,6 \mathrm{a}$ \\
$\mathbf{4 1 7}$ & $3,5 \pm 0,7 \mathrm{~b}$ & $3,5 \pm 0,7 \mathrm{~b}$ & $3,1 \pm 0,7 \mathrm{~b}$ \\
$\mathbf{5 9 8}$ & $4,0 \pm 0,7 \mathrm{~b}$ & $4,6 \pm 0,6 \mathrm{c}$ & $4,0 \pm 0,7 \mathrm{c}$
\end{tabular}

Número de muestras=3; Número de observaciones=45; Número de jueces $=15$

Valores con diferentes letras en la misma columna son estadísticamente diferentes. ( $\mathrm{p}$ valor

$$
<0,05),(\mathrm{n}=3)
$$

354: Tratamiento 1, Excedente de Atún 50\%; 417: Tratamiento 2, Excedente de Atún 70\%; 598 Tratamiento 3, Excedente de Atún 90\%

Se observa en la tabla 5 que en los atributos Olor, Color y Textura aumentan el nivel de acepción a medida que se incrementa el porcentaje del excedente de atún en la formulación. La evaluación sensorial demuestra que las muestras con diferentes porcentajes de excedente si tiene influencia sobre el color y textura; en cambio para el olor se observó que no existe una diferencia significativa en la puntuación de los panelistas para las formulaciones para los tratamiento $2(70 \%)$ y tratamiento $3(90 \%)$. Por otro lado, las muestras que tuvieron menor porcentaje de excedente (50\%) fueron las más bajas en su calificación. 
3. Formulación final del alimento para mascotas

Tabla 6. Formulación final del tratamiento $3(90 \%)$ del alimento para mascota

\begin{tabular}{lr}
\hline Ingrediente & Excedentes del Atún al 90\% \\
\hline Excedente de atún & 38,7 \\
Carne de res molida cocida & 4,3 \\
Almidón de yuca & 10,0 \\
Aislado proteico de soya & 5,0 \\
Sal & 3,0 \\
Ajo en polvo & 3,0 \\
Agua & 25,0 \\
Zanahoria & 10,0 \\
Sorbato de Potasio & 0,4 \\
Ac Cítrico & 0,6 \\
Total & $100 \%$ \\
\hline
\end{tabular}

4. Análisis bromatológicos

A continuación se detallan los resultados de los análisis obtenidos del tratamiento 3 (90\%de excedente) realizados por el laboratorio AVVE S.A.

Tabla 7. Resultados de los análisis químicos del tratamiento 3 (90\% excedente de atún). (LABORATORIOS AVVE S.A, 2017)

\begin{tabular}{ccccc}
\hline \multicolumn{5}{c}{ Resultados Análisis Químicos } \\
\hline Temperatura & $22^{0} \mathrm{C}-33^{0} \mathrm{C}$ & \multicolumn{2}{c}{ Humedad Relativa } & $24 \%-62 \%$ \\
\hline Parámetro & Unidad & Resultados & Requisitos & $\begin{array}{c}\text { Método de } \\
\text { referencia }\end{array}$ \\
Grasa & $\mathrm{g} / 100 \mathrm{~g}$ & 4,38 & - & AOAC 20TH 948.15 \\
Proteínas & $\mathrm{g} / 100 \mathrm{~g}$ & 16,61 & - & AOAC 20TH 940.25 \\
Fósforo & $\mathrm{mg} / 100 \mathrm{~g}$ & 150,98 & - & MMQ-78 \\
Fibra & $\mathrm{g} / 100 \mathrm{~g}$ & 2,54 & - & INEN NTE 0542 \\
Calcio & $\mathrm{mg} / 100 \mathrm{~g}$ & 25,42 & - & MMQ-AAS-18 \\
\hline
\end{tabular}

Tabla 8: Resultados de los análisis microbiológicos del tratamiento 3 (90\% excedente de atún). (LABORATORIOS AVVE S.A, 2017)

\section{Resultados Análisis Microbiológicos}

\begin{tabular}{ccccc}
\hline \multicolumn{4}{c}{ Resultados Análisis Microbiológicos } \\
\hline Temperatura & $18^{\circ} \mathrm{C}-25^{\circ} \mathrm{C}$ & Humedad Relativa & $40 \%-55 \%$ \\
\hline Salmónella spp & $\mathrm{g} / 25 \mathrm{~g}$ & No detectado & No detectado & $\begin{array}{c}\text { METÉtodo de } \\
\text { referencia } \\
\text { MOLECULÁN } \\
\text { AOAC 031208 }\end{array}$ \\
\hline
\end{tabular}

5. Comparación de los resultados

Comparación de los resultados en la formulación final del producto de mayor aceptabilidad t3 (90\%) con respecto a la normativa COVENIN 188883. 
Tabla 9. Comparación del tratamiento 3 respecto a la normativa. (De la A, et al. 2017)

\begin{tabular}{lcc} 
Parámetros & $\begin{array}{c}\text { Norma COVENIN } \\
\mathbf{1 8 8 8 - 8 3}\end{array}$ & $\begin{array}{c}\text { Análisis de } \\
\text { laboratorio }\end{array}$ \\
\hline Humedad \% & 35,0 & $24-62$ \\
Grasa \% & 4,2 & 4,80 \\
Fibra \% & 4,5 & 2,60 \\
Proteína \% & 22,0 & 17,00 \\
Fósforo \% & 0,5 & 0,20 \\
Calcio \% & 1,3 & 0,03 \\
\hline
\end{tabular}

En la tabla 9 se comparan los parámetros nutricionales del alimento para mascota con excedente de atún al $90 \%$. Se observa en los análisis realizados en el laboratorio certificado que existe una aproximación en los resultados nutricionales con respecto a la norma de estudio, mediante el cual, el tratamiento 3 (90\% de excedente de atún) cumple los requisitos específicos requeridos por la norma COVENIN 1888-83 en la elaboración de un alimento completo para felinos; en donde se obtuvo los siguientes resultados: De acuerdo a COVENIN 1888-83 el contenido máximo requerido de calcio es de $1,3 \%$, del cual se obtuvo $0,025 \%$; con respecto al fósforo el contenido mínimo requerido según COVENIN 1888-83 es de 0,5\%, alcanzando un resultado de $0,15 \%$; Asimismo, grasa cruda: el contenido mínimo es $4,5 \%$ según COVENIN 1888-83, el resultado que se obtuvo fue $4,3 \%$; referente a la proteína: la COVENIN 1888-83 establece el contenido mínimo de 22,0\% y el resultado fue 16,61\%; En cuanto a la Fibra cruda: el contenido máximo de fibra requerida es 4,5\% según la COVENIN 1888-83 y se obtuvo 2,5\%; estos valores hacen referencia a la incidencia positiva presente en el porcentaje de excedente de atún mediante su aporte nutricional; cumpliendo los requisitos físicos (Olor y Color) y específicos de acuerdo a la norma de estudio para la elaboración de alimento para mascotas (felinos).

El proyecto de investigación realizado se basó en el desarrollo de un alimento para mascotas utilizando los excedentes del atún garantizando sus propiedades fisicoquímicas y nutricionales. (Oeterrer, 2004) menciona que la industria pesquera genera una gran cantidad de desechos debido a la falta de conocimientos de este recurso como materia prima y fuente para otro producto. Con ello se dio una alternativa para el uso del excedente de atún, así disminuyendo el impacto ambiental que generan las empresas ecuatorianas.

(Kubtza, 2006) indica que en las etapas de procesamiento del pescado los residuos generados: piel, vísceras, esqueleto con carne adherida las cuales representan del $8 \%$ al $16 \%$ de eviscerado y del $60 \%$ al $72 \%$ en producción de filete de piel. En la experimentación realizada aprovechamos estos porcentajes que se generan en la etapa de fileteo del atún, la cual fue empleada como 
materia prima en la formulación y desarrollo de un subproducto a base de excedentes que resulte agradable para las mascota (felinos).

La información proporcionada por (Parrin, 1997) señala que el ensilado de pescado son utilizados para alimentar toda clases de especies animales tales como: animales de piles, rumiantes, cerdos, pollos, peces y mascotas. El desarrollo del alimento para mascota hace referencia favorable en el aprovechamiento de los excedentes del atún innovando en la elaboración de un nuevo producto.

\section{Conclusiones}

El desarrollo de un alimento para mascotas aprovechando los excedentes del atún que se constituirán en materia prima para elaborar un subproducto, será una alternativa rentable para las empresas atuneras ecuatorianas que se beneficiarán mejorando sus ingresos económicos y de la misma manera contribuirán con el cuidado del medio ambiente elaborando un nuevo producto a base de excedentes de atún evitando que estos regresen al mar reduciendo el impacto ambiental.

Los resultados obtenidos en las pruebas sensoriales aplicadas a los usuarios la mejor formulación para la elaboración del alimento de mascotas fue la del tratamiento $3(90 \%)$ de excedente de atún, el cual fue superior a los demás tratamientos dando aceptabilidad del producto por su olor, color y textura compatibles con los resultados en la degustación de las mascotas (felinos) donde se observó un grado de aceptación para la formulación del $90 \%$ (598), debido a que la composición del producto posee mayor cantidad de excedentes de atún en comparación con los tratamiento 1 y tratamiento 2.

Se puede concluir que los análisis bromatológicos realizados en el laboratorio certificado al producto de mayor aceptabilidad seleccionado el tratamiento 3 (90\% de excedentes de atún) se encuentran dentro de los rangos establecidos a la normativa de estudio COVENIN 1888-83, asimismo se determinó que los resultados obtenidos con respecto al calcio, fósforo, proteína, grasa y fibra cruda se encuentran dentro de los parámetros exigidos por el COVENIN 1888-83, estos valores hacen referencia a la incidencia positiva presente en el porcentaje de excedente de atún mediante su aporte nutricional; cumpliendo los requisitos físicos (Olor y Color) y específicos de acuerdo a la norma de estudio para la elaboración de alimento para mascotas (felinos).

\section{References:}

1. Alvarado, C. (2003). Evaluación de alimentos secos para perros (Canis familiaris) en etapa de crecimiento a través de su composición química. Universidad Austral de Chile. Valdivia. Chile. 
2. Bello, R. (1997). Experiencias con Ensilado de Pescado en Venezuela. Tratamiento y utilización de residuos de origen animal, pesquero y alimenticio en la alimentación animal. FAO Library. Caracas.

3. BRISTHAR. (2010). Aditivos alimenticios. Sorbato de Potasio. Disponible en: http://www.bristhar.com.ve/sorbato.html.

4. Calderón, P. (2014). La Sustentabilidad de las Exportaciones de Atún Ecuatoriano: 2014-2020. Universidad de Guayaquil. Guayaquil, Ecuador.

5. Case et al, C. H. (2001). Nutrición Canina y Felina. Guía para profesionales de los animales de compañía. 2a ed. España. Madrid.

6. CLETRAX. (2016). Tecnología Y Líneas. Líneas de producción de alimentos y golosinas para mascotas. Elaboración de alimentos para mascotas.

7. COVENIN. (2017). Alimento completo para caninos y felinos. Norma Venezolana COVENIN 1888-83. Disponible en linea: http://www.sencamer.gob.ve/sencamer/normas/1888-83.pdf .

8. COVENIN1156. (2017). Alimentos para Animales. Determinación de Humedad. Norma Venezolana COVENIN 115-79. Disponible en: http://www.sencamer.gob.ve/sencamer/normas/1156-79.pdf.

9. COVENIN1162. (2017). Alimentos para animales. Determinación de Grasa Cruda. Norma Venezolana COVENIN 1162-79. Disponible en línea en: http://www.sencamer.gob.ve/sencamer/normas/1162-79.pdf.

10. COVENIN1178. (2017). Alimentos. Determinación de Fósforo. Norma Venezolana COVENIN 1178-83. Disponible en línea: http://www.sencamer.gob.ve/sencamer/normas/1178-83.pdf.

11. COVENIN1194. (2017). Alimentos para animales. Determinación de Fibra Cruda. Norma Venezolana COVENIN 1194-79. Disponible en línea:http://www.sencamer.gob.ve/sencamer/normas/1194-79.pdf.

12. COVENIN1195. (2017). Alimentos. Determinación de Nitrogeno. Método de Kjeldahl. Norma Venezolana COVENIN 1195-80. Dispnible en línea: http://www.sencamer.gob.ve/sencamer/normas/1195-80.pdf.

13. COVENIN1291. (2017). Alimentos, Aislamiento e Identificaión de Salmonella. Norma Venezolana COVENIN 1291-88. Disponible en línea: http://www.sencamer.gob.ve/sencamer/normas/1291-88.pdf.

14. COVENIN986. (2017). Alimentos. Determinación de Calcio y Magnesio. Norma Venezolana COVENIN 986-82. Disponible en línea: http://www.sencamer.gob.ve/sencamer/normas/986-82.pdf.

15. Crane, S. G. (2000). Introducción a los alimentos comerciales para mascotas. Nutrición Clínica en Pequeños Animales. 4a ed. Buenos Aires, Argentina. Inter - Medical, 127 - 147. 
16. Donoso, A. (2001). Estudio de la Calidad de los Alimentos Balanceados para caninos de venta en la ciudad de Guayaquil. Universidad de Guayaquil, Ecuador.

17. Eroski. (2016). Pescados y mariscos. Guía práctica sobre pescados. Discponible en: http://pescadosymariscos.consumer.es/propiedadesnutritivas.

18. Gallardo et al, G. M. (2010). Aprovechamiento y transformación de subproductos de la pesca: hacia un manejo sustentable de recursos costeros. Fundación Produce Yucatán.

19. Fueltala, .. (2013). Sector del atún en conserva de Ecuador. Disponible en: http://es.slideshare.net/pafuespi/sector-del-atun-en-conserva-enecuador.

20. Genovese \& Mejía, M. (2010). Creación de una empresa destinada a la producción de alimento balanceado para perros para la cuidad de Guayaquil. Escuela Superior Politécnica del Litoral. Guayaquil.

21. Guerrero, C. (2011). Sector atunero ecuatoriano comercialización de las conservas de atún durante el periodo 2007-2010. Universidad de Guayaquil.

22. Hramann, I. (2010). Aprovechamiento de la Carne Negra de Thunnus Alalunga como Sustituto de Carne Blanca de Pescado en la Elaboración de Paté. Escuela Superior Politécnica del Litoral. Guayaquil. Ecuador.

23. INEN. (2014). Reglamento Técnico ecuatoriano RTE INEN 187. Alimentos para animales de compañía. Intituto Ecuatoriano de normalización.

24. Kubtza, F. (2006). Aproveitamento dos subprodutos do processamento de pescados. Panorama da Aquicultura 16 (94): 23-29.

25. Larose, C. (16 de 05 de 2007). Reliable Palatability Measurement. Recuperado el 12 de 08 de 2017, de key to developing the best test protocols for pet treats: https://www.petfoodindustry.com/articles/620-reliable-palatabilitymeasurement

26. MAKYMAT. (2016). Fuente de proteína: Aislado de Soya. Disponible en:

http://www.makymat.com/contenido/archivospdf/AisladodeSoya.pdf. Guadalajara.

27. Martínez Aispuro, J. A. SUSTITUCIÓN DE ACEITE DE SOYA POR ACEITE DE ATÚN EN LA DIETA DE POLLOS COMO ALTERNATIVA PARA ENRIQUECER LA CARNE CON ÁCIDOS GRASOS OMEGA-3. Interciencia;Caracas;Biblioteca virtual

28. Oeterrer, M. (2004). Tecnologias emergentes para preocessamento do pescado produzido em piscicultura. Páginas 481-50. Brasil. Sao Paulo. 
29. Parín, M. Z. (1997). Aspectos Económicos del Procesamiento y Uso de Ensilado de Pescado. Tratamiento y utilización de residuos de origen animal, pesquero y alimenticio en la alimentación animal. FAO Library. Mar del Plata, Argentina.

30. Pérez \& Zamora, S. (2002). Nutrición y alimentación humana. Universidad de Murcia, 134-135. Murcia.

31. Souza, M. (2003). Processamento do Filé e da pele da Tilápia do Nilo(Oreochromis Niloticus): Aspectos Tecnológicos, Universidade Estadual Paulista, Jaboticabal, SP.

32. Thomas et al, D. S. (2002). Manual de Nutrición y Alimentación en Pequeños Animales. Ediciones S. Barcelona.

33. Villegas, V. M. (2014). Exportaci'n de atún como producto tradicional del Ecuador y su posicionamiento en la unión europea periodo 20092013. Guayaquil. 